\title{
Opioid Prescribing Rates in Nonmetropolitan and Metropolitan Counties Among Primary Care Providers Using an Electronic Health Record System - United States, 2014-2017
}

\begin{abstract}
Macarena C. García, DrPH${ }^{1}$; Charles M. Heilig, $\mathrm{PhD}^{1}$; Scott H. Lee, $\mathrm{PhD}^{1}$; Mark Faul, $\mathrm{PhD}^{2}$; Gery Guy, $\mathrm{PhD}^{2}$; Michael F. Iademarco, MD${ }^{1}$; Katherine Hempstead, $\mathrm{PhD}^{3}$; Dorrie Raymond, $\mathrm{MA}^{4}$; Josh Gray, $\mathrm{MBA}^{4}$
\end{abstract}

Drug overdose is the leading cause of unintentional injuryassociated death in the United States. Among 70,237 fatal drug overdoses in 2017, prescription opioids were involved in $17,029(24.2 \%)(1)$. Higher rates of opioid-related deaths have been recorded in nonmetropolitan (rural) areas (2). In 2017, 14 rural counties were among the 15 counties with the highest opioid prescribing rates.* Higher opioid prescribing rates put patients at risk for addiction and overdose (3). Using deidentified data from the Athenahealth electronic health record (EHR) system, opioid prescribing rates among 31,422 primary care providers ${ }^{\dagger}$ in the United States were analyzed to evaluate trends from January 2014 to March 2017. This analysis assessed how prescribing practices varied among six urban-rural classification categories of counties, before and after the March 2016 release of CDC's Guideline for Prescribing Opioids for Chronic Pain (Guideline) (4). Patients in noncore (the most rural) counties had an $87 \%$ higher chance of receiving an opioid prescription compared with persons in large central metropolitan counties during the study period. Across all six county groups, the odds of receiving an opioid prescription decreased significantly after March 2016. This decrease followed a flat trend during the preceding period in micropolitan and large central metropolitan county groups; in contrast, the decrease continued previous downward trends in the other four county groups. Data from EHRs can effectively supplement traditional surveillance methods for monitoring

\footnotetext{
*U.S. Opioid Prescribing Rate Maps. https://www.cdc.gov/drugoverdose/maps/ rxrate-maps.html.

$\dagger$ Primary care providers in an ambulatory setting; limited to family medicine, family practice, or general practice, or providers who have an internal medicine specialty with no subspecialty. Nurse practitioners and physician assistants are included among primary care providers.
}

trends in opioid prescribing and other areas of public health importance, with minimal lag time under ideal conditions. As less densely populated areas appear to indicate both substantial progress in decreasing opioid prescribing and ongoing need for reduction, community health care practices and intervention programs must continue to be tailored to community characteristics.

Athenahealth is a commercial vendor and developer of cloudbased practice management and EHR systems for physician practices and hospitals. Approximately 100,000 health providers, serving about 86 million patients in the United States, use Athenahealth's applications. This retrospective study used deidentified Athenahealth EHR prescription data from 31,422 primary health care providers serving approximately 17 million
INSIDE
31 Gastroschisis Trends and Ecologic Link to Opioid Prescription Rates — United States, 2006-2015
37 Overdose Deaths Involving Fentanyl and Fentanyl Analogs - New York City, 2000-2017
41 Notes from the Field: Fentanyl Drug Submissions - United States, 2010-2017
44 Notes from the Field: Typhoid Fever Outbreak - Harare, Zimbabwe, October 2017-February 2018
46 Notes from the Field:Tuberculosis Control in the Aftermath of Hurricane Maria — Puerto Rico, 2017
48 QuickStats
Continuing Education examination available at https://www.cdc.gov/mmwr/cme/conted_info.html\#weekly.

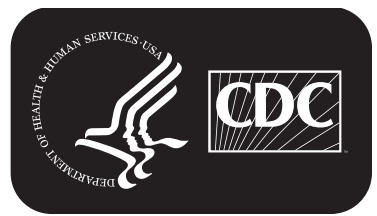


patients. Patient-level data were aggregated by week over the 166 weeks from January 5, 2014, through March 11, 2017. For each week during which a patient had at least one Athenahealth record, that patient contributed one patient-week to this analysis. For each patient-week, it was noted whether primary care providers using Athenahealth's EHR system prescribed one or more opioids (Supplementary Table 1, https://stacks.cdc.gov/ view/cdc/61743). ${ }^{\$}$ Percentage of patient-weeks during which an opioid prescription was written was considered equivalent to the percentage of patients receiving an opioid prescription during that time.

For comparisons over time, data were divided into three periods. Period 1 comprises 52 weeks from January 5, 2014, through January 3, 2015; period 2 includes the next 63 weeks, ending March 19, 2016; and period 3 covers the final 51 weeks, through March 11, 2017. The first cutpoint allows comparisons between the first and second years' data, and the second cutpoint supports comparisons before and after the publication of the CDC Guideline. For comparison by population density, data were stratified by providers' counties according to

\footnotetext{
$\$$ Short and long acting opioid drugs in this study included buprenorphine, butorphanol, codeine, dihydrocodeine, fentanyl, hydrocodone, hydromorphone, levorphanol, meperidine, methadone, morphine, naltrexone, nalbuphine, naloxone, oxycodone, oxymorphone, pentazocine, propoxyphene, tapentadol, and tramadol. The study does not count cough and cold medications containing opioids.
}

CDC's National Center for Health Statistics urban-rural classification scheme. 9 From most to least densely populated, the six categories include large central metropolitan, large fringe metropolitan, medium metropolitan, small metropolitan, micropolitan, and noncore counties.

This analysis includes three components. First, the periodspecific percentage of patients with opioid prescriptions was estimated empirically and with seasonal adjustment using logistic regression. Second, smooth temporal trends were statistically separated from annual seasonal components using locally weighted regression (5). Third, to quantify the period-specific annual rate of increase or decrease in prescribing rates, a second logistic regression model estimated the seasonally adjusted annual percent change (APC) in the odds of receiving an opioid prescription. Statistical software was used for all analyses; statistical tests and confidence intervals (CIs) are presented as simultaneous procedures adjusted for multiple comparisons.

Overall, 128,194,491 patient-weeks of data are included in the analysis; at least one opioid was prescribed during $8,810,237(6.9 \%)$ of these patient-weeks, decreasing from $7.4 \%$ during period 1 to $6.4 \%$ during period 3 (Table 1) (Supplementary Table 2, https://stacks.cdc.gov/view/ cdc/61744). Buprenorphine prescribed for pain and opioid use disorder treatment represented only $0.02 \%$ of all opioid

\footnotetext{
Sttps://www.cdc.gov/nchs/data_access/urban_rural.htm; https://www.cdc.gov/ nchs/data/series/sr_02/sr02_166.pdf.
}

The MMWR series of publications is published by the Center for Surveillance, Epidemiology, and Laboratory Services, Centers for Disease Control and Prevention (CDC), U.S. Department of Health and Human Services, Atlanta, GA 30329-4027.

Suggested citation: [Author names; first three, then et al., if more than six.] [Report title]. MMWR Morb Mortal Wkly Rep 2019;68:[inclusive page numbers].

\section{Centers for Disease Control and Prevention \\ Robert R. Redfield, MD, Director \\ Anne Schuchat, MD, Principal Deputy Director \\ Leslie Dauphin, PhD, Acting Associate Director for Science \\ Barbara Ellis, PhD, MS, Acting Director, Office of Science Quality \\ Chesley L. Richards, MD, MPH, Deputy Director for Public Health Scientific Services \\ Michael F. Iademarco, MD, MPH, Director, Center for Surveillance, Epidemiology, and Laboratory Services}

MMWR Editorial and Production Staff (Weekly)

Charlotte K. Kent, PhD, MPH, Acting Editor in Chief, Executive Editor

Jacqueline Gindler, MD, Editor

Mary Dott, MD, MPH, Online Editor

Teresa F. Rutledge, Managing Editor

Douglas W. Weatherwax, Lead Technical Writer-Editor

Glenn Damon, Soumya Dunworth, PhD, Teresa M. Hood, MS, Technical Writer-Editors

Matthew L. Boulton, MD, MPH Virginia A. Caine, MD

Katherine Lyon Daniel, PhD

Jonathan E. Fielding, MD, MPH, MBA

David W. Fleming, MD

William E. Halperin, MD, DrPH, MPH
MMWR Editorial Board Timothy F. Jones, MD, Chairman Robin Ikeda, MD, MPH Phyllis Meadows, PhD, MSN, RN Jewel Mullen, MD, MPH, MPA Jeff Niederdeppe, $\mathrm{PhD}$ Patricia Quinlisk, MD, MPH
Martha F. Boyd, Lead Visual Information Specialist Maureen A. Leahy, Julia C. Martinroe, Stephen R. Spriggs, Tong Yang, Visual Information Specialists Quang M. Doan, MBA, Phyllis H. King, Terraye M. Starr, Moua Yang, Information Technology Specialists
Stephen C. Redd, MD,

Patrick L. Remington, MD, MPH

Carlos Roig, MS, MA

William Schaffner, MD

Morgan Bobb Swanson, BS 
TABLE 1. Number and percentage of patient-weeks with at least one opioid prescription - Athenahealth, United States, January 2014March 2017

\begin{tabular}{|c|c|c|c|c|c|c|}
\hline \multirow[b]{2}{*}{ Urban-rural category* } & \multirow[b]{2}{*}{ No. of patient-weeks } & \multirow{2}{*}{$\begin{array}{l}\text { No. receiving opioid } \\
\text { prescription }\end{array}$} & \multicolumn{4}{|c|}{ Percentage receiving opioid prescription } \\
\hline & & & Overall & Period $1^{\dagger}$ & Period $2^{\dagger}$ & Period $3^{\dagger}$ \\
\hline Noncore & $8,979,403$ & 864,364 & 9.6 & 10.3 & 9.9 & 9.0 \\
\hline Micropolitan & $16,342,824$ & $1,532,747$ & 9.4 & 9.4 & 9.6 & 9.1 \\
\hline Small metro & $18,860,569$ & $1,443,246$ & 7.7 & 8.0 & 7.7 & 7.4 \\
\hline Medium metro & $32,045,592$ & $2,158,111$ & 6.7 & 7.3 & 6.9 & 6.2 \\
\hline Large fringe metro & $31,430,958$ & $1,753,802$ & 5.6 & 6.4 & 5.8 & 5.0 \\
\hline Large central metro & $20,535,145$ & $1,057,967$ & 5.2 & 5.4 & 5.2 & 5.0 \\
\hline All counties & $128,194,491$ & $8,810,237$ & 6.9 & 7.4 & 7.0 & 6.4 \\
\hline
\end{tabular}

* National Center for Health Statistics urban-rural classification scheme for counties. https://www.cdc.gov/nchs/data_access/urban_rural.htm.

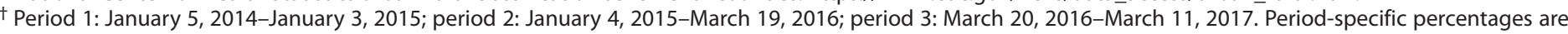
based on raw counts rather than statistical models.

prescriptions. By county classification, the overall percentage of patients with opioid prescriptions ranged from $5.2 \%$ in large central metropolitan counties to $9.6 \%$ in noncore counties during the study period. Patients in noncore counties had an $87 \%$ higher chance of receiving an opioid prescription than did patients in large central metropolitan areas during the study period.

The lowest period-specific percentages of patient-weeks with an opioid prescription occurred in large central metropolitan counties $(5.0 \%-5.4 \%)(\mathrm{p}<0.001$, multiplicity-adjusted Wald tests), except during period 3, when percentages in large metropolitan counties (5.0\%) were the same as those in large fringe metropolitan counties (5.0\%) (Supplementary Table 2, https://stacks.cdc.gov/view/cdc/61744). In contrast, the highest period-specific percentages $(9.0 \%-10.3 \%)$ were in noncore counties $(\mathrm{p}<0.02)$, except in period 3 , when percentages in noncore counties $(9.0 \%)$ were similar to those in micropolitan counties (9.1\%). Across metropolitan and nonmetropolitan categories, all percentages of weeks with an opioid prescription during period 2 were significantly different from those in period 1 , and percentages in period 3 differed significantly from those in period $2(\mathrm{p}<0.003)$.

Visual inspection of the prescribing trends by urbanrural status and by period revealed patterns in both the raw (Supplementary Figure 1, https://stacks.cdc.gov/view/ cdc/61741) and seasonally adjusted (Supplementary Figure 2, https://stacks.cdc.gov/view/cdc/61742) data. During period 1, before release of the CDC Guideline, the odds of receiving an opioid prescription increased $6.4 \%$ per year in noncore counties (95\% multiplicity-adjusted Wald CI $=2.1-10.8$ ), and $9.7 \%$ per year in micropolitan counties $(95 \% \mathrm{CI}=6.5-$ 13.0) (Table 2) (Figure). During period 3, after release of the CDC Guideline, the odds of receiving an opioid prescription decreased significantly in all county groups. Comparing trends between periods, the APC increased in large central metropolitan counties in period 2 compared with period 1 $(\mathrm{p}<0.001)$ and decreased between periods 2 and $3(\mathrm{p}<0.001)$. In the other five urban-rural categories, the APC decreased in period 2 compared with period $1(\mathrm{p}<0.02)$; among these five groups, only micropolitan counties experienced a significant decrease in APC between periods 2 and $3(\mathrm{p}<0.001)$.

TABLE 2. Annual percent change (APC) in odds of receiving at least one opioid prescription - Athenahealth, United States, January 2014-March 2017

\begin{tabular}{|c|c|c|c|c|c|}
\hline \multirow[b]{2}{*}{ Urban-rural category* } & \multirow{2}{*}{$\frac{\text { Period } 1^{\dagger}}{\operatorname{APC}(95 \% \mathrm{CI})}$} & \multirow{2}{*}{$\frac{\text { Period } 2^{\dagger}}{\operatorname{APC}(95 \% \mathrm{CI})}$} & \multirow{2}{*}{$\frac{\text { Period } 3^{\dagger}}{\operatorname{APC}(95 \% \mathrm{CI})}$} & \multicolumn{2}{|c|}{ p-value (direction of change) ${ }^{\S}$} \\
\hline & & & & Period 1 versus period 2 & Period 2 versus period 3 \\
\hline Noncore & $6.4(2.1 \text { to } 10.8)^{9}$ & $-10.1(-12.2 \text { to }-8.0)^{9}$ & $-7.5(-10.7 \text { to }-4.2)^{9}$ & $<0.001$ (decrease) & $0.713(-)$ \\
\hline Micropolitan & $9.7(6.5 \text { to } 13.0)^{9}$ & $-0.8(-2.6$ to 0.9$)$ & $-13.3(-15.6 \text { to }-10.9)^{9}$ & $<0.001$ (decrease) & $<0.001$ (decrease) \\
\hline Small metro & $0.2(-2.8$ to 3.2$)$ & $-4.5(-6.2 \text { to }-2.7)^{9}$ & $-5.8(-8.4 \text { to }-3.2)^{9}$ & 0.013 (decrease) & $0.977(-)$ \\
\hline Medium metro & $-2.5(-4.8 \text { to }-0.1)^{* *}$ & $-8.7(-10.1 \text { to }-7.4)^{9}$ & $-9.2(-11.2 \text { to }-7.2)^{9}$ & $<0.001$ (decrease) & $0.999(-)$ \\
\hline Large fringe metro & $-2.0(-4.7$ to 0.8$)$ & $-14.9(-16.2 \text { to }-13.5)^{9}$ & $-13.1(-15.1 \text { to }-10.9)^{9}$ & $<0.001$ (decrease) & $0.616(-)$ \\
\hline Large central metro & $-9.9(-13.2 \text { to }-6.4)^{9}$ & $1.8(-0.3$ to 3.9$)$ & $-11.7(-14.3 \text { to }-8.9)^{9}$ & $<0.001$ (increase) & $<0.001$ (decrease) \\
\hline All counties & $-1.4(-10.6$ to 8.8$)$ & $-8.2(-13.3 \text { to }-2.7)^{\dagger+}$ & $-10.4(-17.9 \text { to }-2.2)^{t \dagger}$ & $0.371(-)$ & $0.856(-)$ \\
\hline
\end{tabular}

Abbreviation: $\mathrm{Cl}=$ confidence interval.

* National Center for Health Statistics urban-rural classification scheme for counties. https://www.cdc.gov/nchs/data_access/urban rural.htm.

† Period 1: January 5, 2014-January 3, 2015; period 2: January 4, 2015-March 19, 2016; period 3: March 20, $2016-$ March 11 , 2017.

$\S p$-values from multiplicity-adjusted Wald tests; $(-)$ indicates a nonsignificant difference $(p>0.05)$ between APCs in adjacent periods.

१ $p<0.001$.

** $p<0.05$.

†† $\mathrm{p}<0.01$ 
FIGURE. Model-based trends in percentage of patient-weeks with at least one opioid prescription, by urban-rural category - Athenahealth, United States, January 2014-March 2017

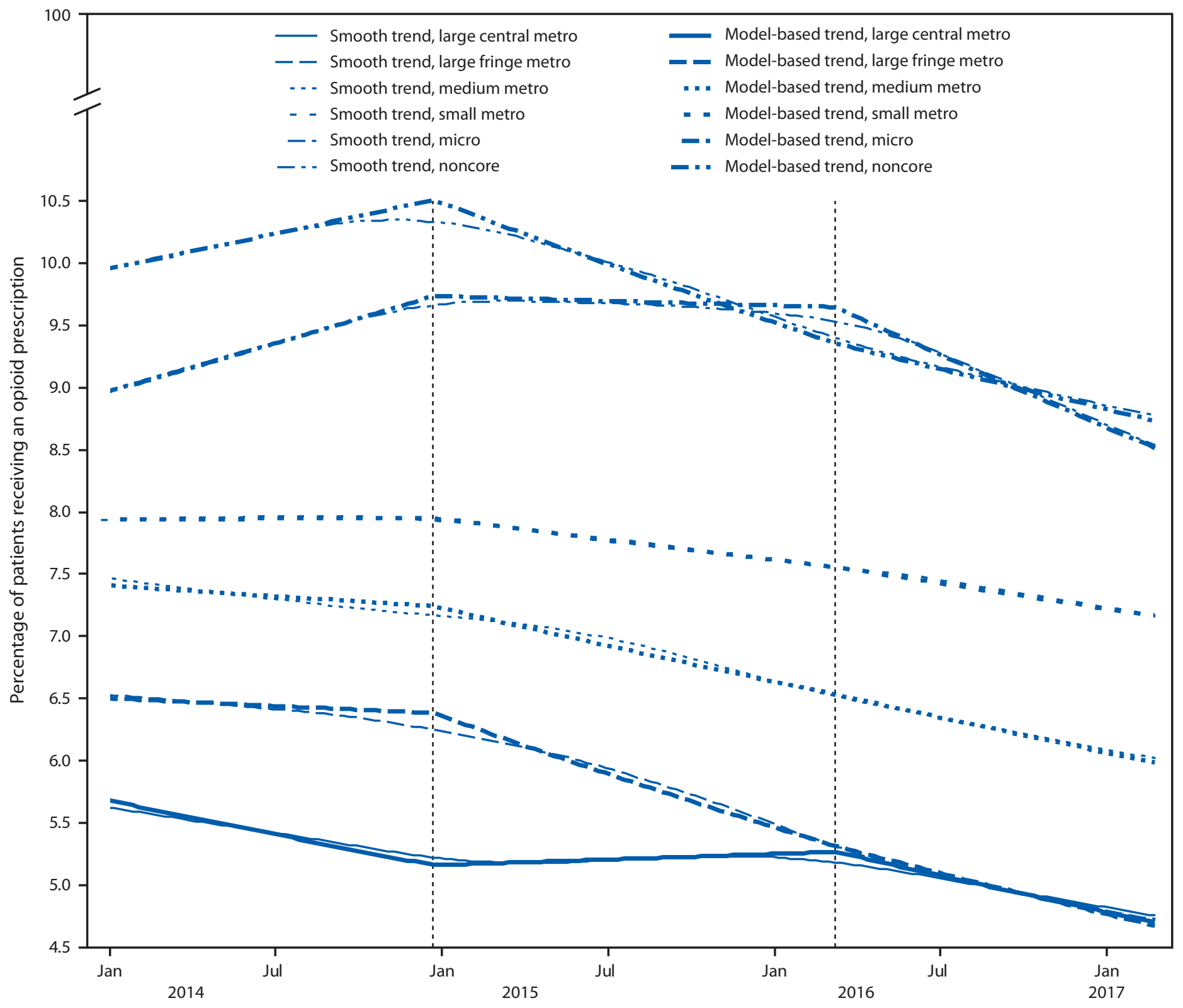

Date of prescription

\section{Discussion}

Throughout the analysis period, opioid prescribing rates by primary care providers were significantly higher in nonmetropolitan counties than in metropolitan counties. Whereas the prescribing rate increased from January 2014 through January 2015 (period 1) in both micropolitan and noncore counties, those trends halted, and rates became flat or declined through mid-March 2016 (period 2). Trends in all other urban-rural categories were flat or decreasing over the same two periods. The odds of a patient receiving an opioid prescription decreased in all urban-rural county groups after the March 2016 publication of the CDC Guideline. Those trends represented significant decreases in the micropolitan and large central metropolitan categories. In the other four county groups, however, the significant decreases after March 2016 represented a continuation of previously decreasing trends.

Higher odds of opioid prescribing in nonmetropolitan counties might be attributed in part to prescription drug use and misuse at an earlier age as well as higher prevalences of chronic pain among persons living in rural areas $(6,7)$. Nonmetropolitan 
counties also tend to have larger populations of older adults who have higher prevalences of conditions associated with pain $(6)$. Opioid prescribing in rural (nonmetropolitan) areas is strongly influenced by providers' individual relationships with their patients (8), and can be inconsistent with opioid prescribing guidelines. As well, access to medication-assisted treatment facilities and alternative therapies are limited in rural areas (8). Variations in the implementation of state-run prescription drug monitoring programs and state-based laws (9), such as the regulation of pain-management clinics, might also differ in urban and rural communities.

Despite reductions in opioid prescribing in recent years (1), opioid-involved overdose death rates have increased, largely driven by heroin and illicitly manufactured fentanyl (2). Many persons who self-report heroin use have a history of misusing prescription opioids (10). Addressing prescription opioid use is an important step in curbing opioid-involved overdose deaths. Interventions such as using Prescription Drug Monitoring Programs and practices that align with evidencebased adoption of the CDC Guideline can improve prescribing decisions. ${ }^{* *}$ The Guideline can help providers and patients weigh the benefits and risks of prescribing opioids according to best available evidence and individual patient needs (4). This study demonstrates that data from EHRs can effectively supplement traditional surveillance methods for monitoring trends in opioid prescribing and other areas of public health importance. The lag between the collection of the data and this analysis could potentially be reduced to a matter of weeks with optimized workflows.

The findings in this report are subject to at least three limitations. First, the conclusions drawn from the records provided by Athenahealth might not be generalizable to all patients in primary care. Second, although the data include all patients with an opioid prescription, they do not include other characteristics of each prescription, including indication (e.g., chronic versus acute pain or opioid use disorder treated with buprenorphine [although this drug accounted for a small fraction of all opioids prescribed]) and whether prescriptions were filled and taken as prescribed. Finally, this analysis does not account for differing demographic profiles across counties, such as age distributions and payer types, which could be confounded by population density in its association with opioid prescribing rates.

The percentage of patients who received an opioid prescription was lower in more densely populated counties than among less populated rural counties; however, all areas, including rural counties, experienced substantial decreases in prescribing

\footnotetext{
** https://www.cdc.gov/drugoverdose/pdf/prescribing/CDC-DUIP-QualityIm provementAndCareCoordination-508.pdf.
}

\section{Summary}

What is already known about this topic?

Opioid prescribing rates vary by county urbanization level and are declining overall.

What is added by this report?

Analysis of patient opioid prescription data from a national electronic health record vendor during 2014-2017 found that the percentage of patients prescribed an opioid was higher in rural than in urban areas. Significant decreases in opioid prescribing occurred across all urban-rural categories after the March 2016 release of the CDC Guideline for Prescribing Opioids for Chronic Pain.

What are the implications for public health practice?

As less densely populated areas indicate both progress in decreasing opioid prescribing and need for ongoing reduction, tailoring community health care practices and intervention programs to community characteristics will remain important.

over time. As less densely populated areas appear to indicate both substantial progress in decreasing opioid prescribing and ongoing need for reduction, community health care practices and intervention programs must continue to be tailored to community characteristics.

\section{Acknowledgment}

Philip Galebach, formerly of Athenahealth, AthenaResearch, Watertown, Massachusetts.

Corresponding author: Macarena C. García, mcgarcia@cdc.gov, 404-539-4410.

\footnotetext{
${ }^{1}$ Center for Surveillance, Epidemiology, and Laboratory Services, CDC; ${ }^{2}$ National Center for Injury Prevention and Control, CDC; ${ }^{3}$ Robert Wood Johnson Foundation, Princeton, New Jersey; ${ }^{4}$ Athenahealth, AthenaResearch, Watertown, Massachusetts.
}

All authors have completed and submitted the ICMJE form for disclosure of potential conflicts of interest. No potential conflicts of interest were disclosed.

\section{References}

1. Scholl L, Seth P, Kariisa M, Wilson N, Baldwin G. Drug and opioidinvolved overdose deaths_-United States, 2013-2017. MMWR Morb Mortal Wkly Rep 2018;67:1419-27. https://doi.org/10.15585/mmwr. mm675152e1

2. Mack KA, Jones CM, Ballesteros MF. Illicit drug use, illicit drug use disorders, and drug overdose deaths in metropolitan and nonmetropolitan areas-United States. Am J Transplant 2017;17:3241-52. https://doi. org/10.1111/ajt.14555

3. CDC. Vital signs: overdoses of prescription opioid pain relievers—United States, 1999-2008. MMWR Morb Mortal Wkly Rep 2011;60:1487-92.

4. Dowell D, Haegerich TM, Chou R. CDC guideline for prescribing opioids for chronic pain-United States, 2016. MMWR Recomm Rep 2016;65(No. RR-1). https://doi.org/10.15585/mmwr.rr6501e1

5. Cleveland RB, Cleveland WS, McRae JE, Terpenning I. STL: a seasonaltrend decomposition procedure based on loess. J Off Stat 1990;6:3-33. 
6. Keyes KM, Cerdá M, Brady JE, Havens JR, Galea S. Understanding the rural-urban differences in nonmedical prescription opioid use and abuse in the United States. Am J Public Health 2014;104:e52-9. https://doi. org/10.2105/AJPH.2013.301709

7. Monnat SM, Rigg KK. Examining rural/urban differences in prescription opioid misuse among US adolescents. J Rural Health 2016;32:204-18. https://doi.org/10.1111/jrh.12141

8. Click IA, Basden JA, Bohannon JM, Anderson H, Tudiver F. Opioid prescribing in rural family practices: a qualitative study. Subst Use Misuse 2018;53:533-40. https://doi.org/10.1080/10826084.2017.1342659
9. Rutkow L, Chang HY, Daubresse M, Webster DW, Stuart EA, Alexander GC. Effect of Florida's prescription drug monitoring program and pill mill laws on opioid prescribing and use. JAMA Intern Med 2015;175:1642-9. https://doi.org/10.1001/jamainternmed.2015.3931

10. Compton WM, Jones CM, Baldwin GT. Relationship between nonmedical prescription-opioid use and heroin use. N Engl J Med 2016;374:154-63. https://doi.org/10.1056/NEJMra1508490 